\title{
ON THE MECHANISM OF EXCITATION ENERGY TRANSFER INVOLVING LONG AND SHORT RANGE INTERACTION IN DILUTE ORGANIC LIQUID SCINTILLATOR SYSTEMS
}

\author{
S.M. Hanagodimath, G.S. Gadaginamath and G.C. Chikkur \\ Department of Physics, Karnatak University \\ Dharwad 580 003, India
}

(Received May 22, 1990; revised version November 7, 1991)

\begin{abstract}
The rate parameter $K_{3}$ of solvent-solute energy transfer and the rate parameter $K_{7 \mathrm{~b}}$ for solvent-quencher energy transfer are determined experimentally under ultraviolet excitation for a system Ethyl 1 butyl-2-methyl-5 ethoxycarboxy methoxy indole-3-carboxylate (EBMEC) in a deoxygenated system comprising 1:9 mixture of toluene-cyclohexane as a function of temperature in the range of $20-70^{\circ} \mathrm{C}$, using bromobenzene as a solvent quencher. The data is analysed in terms of compact equation formed by combining Voltz et al. and Birks and Conte models. The interaction distance for the energy transfer from the excited solvent to solute molecules and solvent to quencher molecules are determined using this equation. The magnitude of the interaction distance indicates that the excitation energy transfer takes place due to long-range interaction in the case of solvent-solute energy transfer and short-range interaction in the case of solvent quencher energy transfer in dilute systems.
\end{abstract}

PACS numbers: $34.30 .+\mathrm{h}$

\section{Introduction}

It is well-known that in organic liquid scintillators the transfer of excitation energy from solvent to solute molecules is due to (a) Brownian diffusion, (b) energy migration and (c) resonance interaction due to Förster process. Various energy transfer models [1-4] have been suggested to understand the role played by these above-mentioned processes. Voltz et al. [1] have proposed that all the three processes $(a, b, c)$ play an important role, whereas Birks and Conte [2] have proposed that only the diffusion and the migration processes play an important role in the energy transfer from solvent to solute molecules. Other models are also proposed recently [5-7]. The purpose of the present investigation is to determine 
the contribution due to long-range interaction in the energy transfer process using a scintillator system consisting of toluene + cyclohexane (TC) in the ratio of 1:9 with bromobenzene as a quencher and EBMEC as a scintillator (EBMEC belongs to indole series which are known for their fluorescence efficiency). The results of various parameters determined in this study are reported for the solute in a deoxygenated TC solution under ultraviolet excitation in the $20-70^{\circ} \mathrm{C}$ temperature range. The experimental data has been analysed in terms of a compact equation formed by combining. Voltz et al. and Birks and Conte models.

Cyclohexane was chosen to dilute toluene because it is a neutral diluent and does not take part in the energy transfer process. But on the other hand the excited solvent molecules will be surrounded by a large number of neutral diluent molecules due to which the energy migration between the solvent molecules will be hindered. Since the viscosity of the medium does not change much by dilution the diffusion process remains unaffected. Therefore there is a possibility of long-range interaction to play a part to compensate the hindrance caused to the migration process.

\section{Theory}

When ultraviolet radiation of proper wavelength is used to excite the solvent, the solvent molecules get excited to a particular level and transfer part of their excitation energy to the solute molecules which fluoresce. Depending on the nature of the solvent, solute and quencher, the quencher molecule may quench either the excited solvent molecule or the excited solute molecule or both. The phenomenon of energy transfer and quenching in liquid systems can be explained by the following well-known kinetic scheme, in which $\mathrm{X}$ and $\mathrm{Y}$ represent solvent and solute molecules respectively and an asterisk represents the excited state of the molecule, and $K$ 's the corresponding rate parameters.

\begin{tabular}{lllcl}
\hline No & \multicolumn{2}{c}{ Reaction } & $\begin{array}{c}\text { Rate } \\
\text { parameter }\end{array}$ & Process \\
\hline 1 & $\mathrm{X}+h \nu$ & $\ldots \mathrm{X}^{*}$ & $K_{0}$ & Absorption \\
$2 \mathrm{a}$ & $\mathrm{X}^{*}$ & $\ldots \mathrm{X}+h \nu$ & $K_{1}$ & Radiative emission \\
$2 \mathrm{~b}$ & $\mathrm{X}^{*}$ & $\ldots \mathrm{X}$ & & Internal quenching \\
3 & $\mathrm{X}^{*}+\mathrm{X}$ & $\ldots 2 \mathrm{X}$ & $K_{2} \mathrm{X}$ & Concentration quenching \\
4 & $\mathrm{X}^{*}+\mathrm{Y}$ & $\ldots \mathrm{X}+\mathrm{Y}^{*}$ & $K_{3} \mathrm{Y}$ & Nonradiative energy transfer \\
5 & $\mathrm{Y}^{*}+\mathrm{Y}$ & $\ldots 2 \mathrm{Y}$ & $K_{4} \mathrm{Y}$ & Concentration quenching \\
6 & $\mathrm{Y}^{*}$ & $\ldots \mathrm{Y}$ & $K_{5}$ & Internal quenching \\
7 & $\mathrm{Y}^{*}$ & $\ldots \mathrm{Y}+h \nu$ & $K_{6}$ & Fluorescence emission
\end{tabular}

When an external quencher which quenches only the excited solvent molecules is used the following quenching reaction may occur:

$$
\begin{array}{llllll}
8 & \mathrm{X}^{*}+Z_{\mathrm{b}} & \ldots \mathrm{X}+Z_{\mathrm{b}} & K_{7 \mathrm{~b}} Z_{\mathrm{b}} & \text { External quenching }
\end{array}
$$

Here $Z_{\mathrm{b}}$ represents the molecular species of the quencher as well as its concentration. The fluorescence intensity $I$ in the presence of an external quencher of 
concentration $Z_{\mathrm{b}}$ is given by

$$
I=\frac{K_{0} K_{3} Y}{\left(K_{1}+K_{2} X+K_{3} Y+K_{7 \mathrm{~b}} Z_{\mathrm{b}}\right)} \frac{K_{6}}{\left(K_{4} Y+K_{5}+K_{6}\right)}
$$

If $I_{0}$ is the fluorescence intensity in the absence of the external quencher, then the ratio $I_{0} / I$ is given by the Stern-Volmer equation,

where

$$
I_{0} / I=\left(1+\gamma Z_{\mathrm{b}}\right)
$$

$$
\gamma^{-1}=\frac{\left(K_{1}+K_{2} X\right)}{K_{7 \mathrm{~b}}}+\frac{K_{3} Y}{K_{7 \mathrm{~b}}} .
$$

Thus if one measures $I$ as a function of $Z_{\mathrm{b}}$, one can determine from Eq. (2) the quenching constant $\gamma$ at a fixed solute concentration $Y$. Further, from Eq. (3) a plot of $\gamma^{-1}$ versus $Y$ is a straight line with the intercept

$$
i=\left(K_{1}+K_{2} X\right) / K_{7 \mathrm{~b}}
$$

and the slope

$$
s=K_{3} / K_{7 \mathrm{~b}} \text {. }
$$

Using the literature value of $\left(K_{1}+K_{2} X\right)^{-1}$ which is the decay time $\tau_{\mathrm{X}}$ of the solvent in the absence of the solute one can determine $K_{3}$ and $K_{7 \mathrm{~b}}$ from Eqs. (4) and (5) respectively at each temperature.

\section{Results and discussion}

The fluorescence intensity $I$ at different quencher concentrations $Z_{\mathrm{b}}$ (where $Z$ is the quencher concentration and $b$ - the suffix which represents bromobenzene) at a fixed solute concentration $Y$ is measured. The error in the measurement of $I$ is within $5 \%$. The experimental technique and the method of analysis of the data is as described in our earlier paper [8]. The quenching constant $\gamma$ is determined at each temperature using the Stern-Volmer plots. The plots of $\gamma^{-1}$ versus $Y$ at each temperature are shown in Fig. 1. From Fig. 1, we see that $\gamma^{-1}$ varies linearly with $Y$. As discussed earlier [8] we get the values for the quantities $\left(K_{1}+K_{2} X\right) / K_{7 \mathrm{~b}}$ and $K_{3} / K_{7 \mathrm{~b}}$ from the intercepts and slopes respectively from Eqs. (4) and (5). In order to determine $K_{3}$ and $K_{7 \mathrm{~b}}$ we have used the independent value of $\tau_{\mathrm{X}}$ determined by Weinreb [9]. These values are given in the Table.

Voltz et al. [1] proposed an expression for energy transfer process from solvent to solute molecules considering that all the three processes, viz. diffusion, migration and dipole-dipole interaction are playing a part. They gave the expression for the rate parameter $K_{3}$ as:

$$
K_{3}=2 \pi N^{\prime}\left(D_{\mathrm{XY}}+\Lambda\right) R_{0},
$$

where $N^{\prime}$ is the Avogadro number per millimole, $D_{\mathrm{XY}}$ is the sum of the diffusion coefficients $D_{\mathrm{X}}$ and $D_{\mathrm{Y}}$ of the solvent and solute molecules respectively, $\Lambda$ is the energy migration coefficient of the solvent and $R_{0}$ is the Förster critical energy transfer distance.

Birks and Conte [2] proposed an expression for energy transfer process from solvent to solute molecules considering that only diffusion and migration processes are playing a part. They gave the expression for the rate parameter $K_{3}$ as:

$$
K_{3}=4 \pi N^{\prime}\left(D_{\mathrm{XY}}+\Lambda\right) P_{\mathrm{XY}} R_{\mathrm{XY}}
$$




\section{TABLE}

Value of diffusion coefficient of solvent $\left(D_{\mathbf{X}}\right)$, of solute $\left(D_{\mathrm{Y}}\right)$, of quencher $\left(D_{Q}\right)$, the energy transfer rate parameter $K_{3}$, the quenching rate parameter $K_{7 b}$, solvent fluorescence life time $\tau_{\mathbf{X}}$ at different temperatures and the molecular radius $R_{\mathrm{X}}$ of solvent, $R_{\mathrm{Y}}$ of solute, $R_{\mathrm{Q}}$ of quencher, the Förster critical energy transfer distance $R_{0}$, energy migration coefficient $A$ for $K_{3}$ and $K_{7 \mathrm{~b}}$ and effective energy transfer distance $P_{\mathrm{xy}} R_{\text {eff }}$ between solvent and solute, $P_{\mathrm{XQ}} R_{\mathrm{eff}}$ between solvent and quencher.

\begin{tabular}{|c|c|c|c|c|c|c|}
\hline$t\left({ }^{\circ} \mathrm{C}\right)$ & $\begin{array}{c}D_{\mathrm{X}} \\
10^{-5} \\
\mathrm{~cm}^{2} \mathrm{~s}^{-1}\end{array}$ & $\begin{array}{c}D_{\mathrm{Y}} \\
10^{-5} \\
\mathrm{~cm}^{2} \mathrm{~s}^{-1}\end{array}$ & $\begin{array}{c}D_{\mathrm{Q}} \\
10^{-5} \\
\mathrm{~cm}^{2} \mathrm{~s}^{-1}\end{array}$ & $\begin{array}{c}K_{3} \\
10^{10} \\
\mathrm{M}^{-1} \mathrm{~s}^{-1}\end{array}$ & $\begin{array}{c}K_{7 \mathrm{~b}} \\
10^{10} \\
\mathrm{M}^{-1} \mathrm{~s}^{-1}\end{array}$ & $\begin{array}{c}\tau_{\mathrm{X}} \\
10^{-9} \\
\mathrm{~s}\end{array}$ \\
\hline 20 & 0.86 & 0.58 & 0.85 & 3.75 & 0.50 & 28 \\
\hline 30 & 1.08 & 0.72 & 1.03 & 4.12 & 0.54 & 26 \\
\hline 40 & 1.31 & 0.87 & 1.26 & 4.47 & 0.58 & 23 \\
\hline 50 & 1.55 & 1.04 & 1.49 & 4.92 & 0.63 & 20 \\
\hline 60 & 1.84 & 1.23 & 1.77 & 5.43 & 0.69 & 17 \\
\hline 70 & 2.15 & 1.4 & 2.07 & 5.97 & 0.75 & 14 \\
\hline
\end{tabular}

and when an external quencher is added to the scintillator system, they proposed that quenching process is also due to diffusion and migration processes only. And the quenching rate parameter $K_{7 \mathrm{~b}}$ is given by

$$
K_{7 \mathrm{~b}}=4 \pi N^{\prime}\left(D_{\mathrm{XQ}}+\Lambda\right) P_{\mathrm{XQ}} R_{\mathrm{XQ}}
$$

where $D_{\mathrm{XQ}}$ is the sum of the diffusion coefficients $D_{\mathrm{X}}$ and $D_{\mathrm{Q}}$ of the solvent and quencher molecules respectively, $P_{X Y}$ and $P_{X Q}$ are the interaction probabilities per encounter between solvent-solute and solvent-quencher molecules respectively. According to Birks and Conte model the value of $P_{X Y}$ is always taken to be unity and that of $P_{\mathrm{XQ}}$ is taken to be less than unity. The quantity $R_{\mathrm{XY}}$ is the interaction distance between the solvent and solute molecules and $R_{\mathrm{XQ}}$ is that between solvent and quencher molecules.

We have formed [10] a compact equation by combining Eqs. (6), (7) and (8) as:

$$
K_{3} \text { or } K_{7 \mathrm{~b}}=4 \pi N^{\prime}(D+\Lambda) P R_{\mathrm{eff}},
$$

in which $D=D_{\mathrm{XY}}, P=P_{\mathrm{XY}}$ and $R_{\mathrm{eff}}=R_{\mathrm{XY}}$ or $R_{0} / 2$ for $K_{3}$ and $D=D_{\mathrm{XQ}}$, $P=P_{\mathrm{XQ}}$ and $R_{\mathrm{eff}}=R_{\mathrm{XQ}}$ for $K_{\mathrm{7b}}$, which is applicable to both energy transfer and quenching processes.

Now we consider the various parameters occurring in Eq. (9) viz. $D_{\mathbf{X}}, D_{\mathbf{Y}}$ and $D_{\mathrm{Q}}$ the diffusion coefficients of the solvent, solute and quencher molecules 


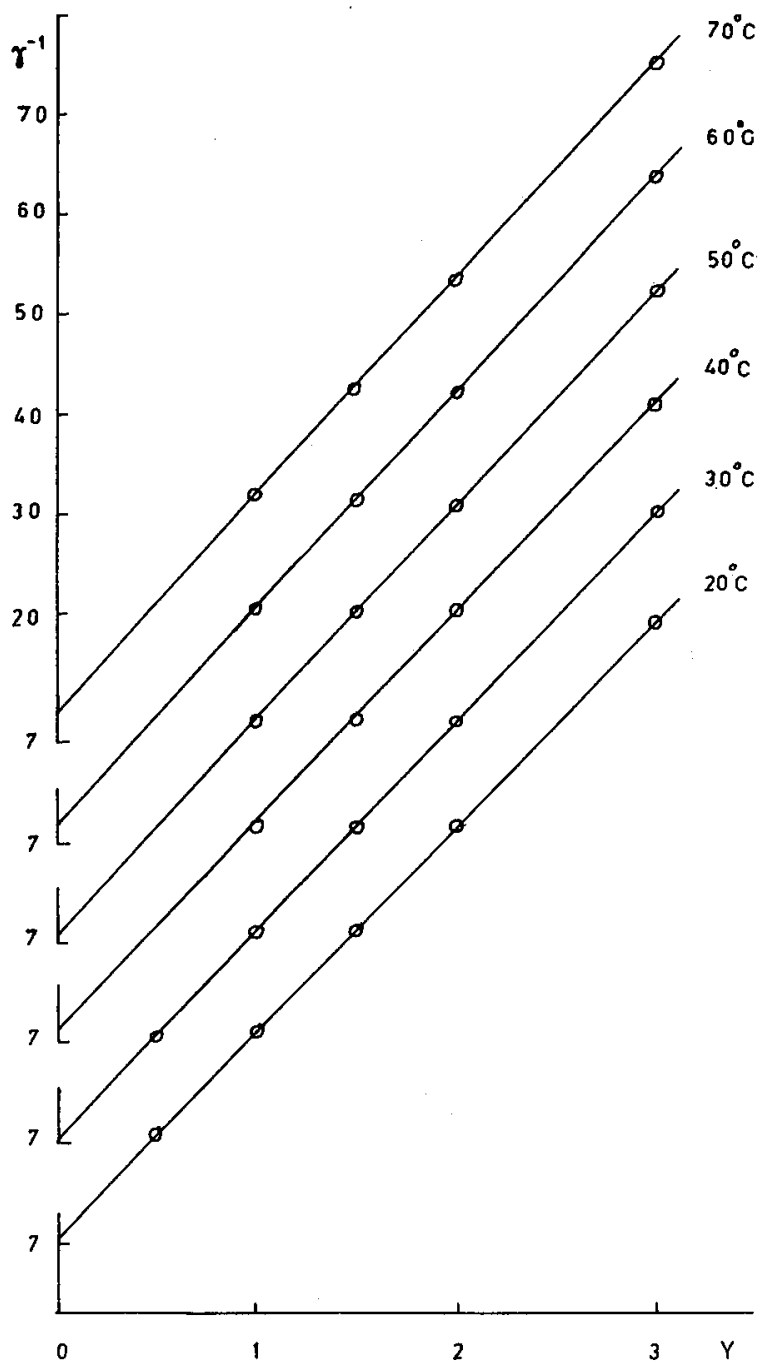

Fig. 1. Variation of $\gamma^{-1}$ (in $10^{-3} \mathrm{~mole} / \mathrm{dm}^{3}$ ) with solute concentration $Y$ (in $\mathrm{g} / \mathrm{dm}^{3}$ ) in the $20-70^{\circ} \mathrm{C}$ temperature range.

respectively, $R_{\mathrm{X}}, R_{\mathrm{Y}}$ and $R_{\mathrm{Q}}$ the radii of the solvent, solute and quencher molecules respectively and $R_{0}$ the Förster critical energy transfer distance.

The self-diffusion coefficients $D_{\mathrm{X}}, D_{\mathrm{Y}}$ and $D_{\mathrm{Q}}$ are calculated using the Stokes-Einstein relation [11]:

$$
D=k T / a \pi \eta R,
$$

where $k$ is the Boltzmann constant, $T$ is the absolute temperature, $\eta$ is the viscosity of the solvent, $R$ is the radius of the diffusing molecule and $a$ is a constant referred to as the Stokes-Einstein number. We have used the experimentally measured values [12] of viscosities of the solvent in the $20-70^{\circ} \mathrm{C}$ temperature range using 
standard Ostwald viscometer. Since the concentrations of the solute and quencher are very low, the viscosity of the solute and quencher are taken to be the same as that of the medium. The radii of the solvent, solute and quencher molecules are calculated by adding the atomic volumes of all the atoms constituting the molecules as suggested by Edward [13], and these values are given in the Table. The Stokes-Einstein number, $a_{\mathrm{X}}=a_{\mathrm{Y}}=a_{\mathrm{Q}}=6$ is chosen since the values of diffusion coefficients determined experimentally agree with the calculated values for $a_{\mathrm{X}}=\dot{a}_{\mathrm{Y}}=a_{\mathrm{Q}}=6$ at different temperatures. The diffusion coefficients thus determined using Eq. (10) at each temperature are given in the Table.

The Förster critical energy transfer distance $R_{0}$ was calculated using the equation [14]

$$
R_{0}^{6}=\frac{9000 \ln (10) \psi^{2} \phi_{x}}{128 \pi^{5} n^{4} N^{\prime}} \int_{0}^{\infty} \frac{f_{x}(\bar{\nu}) \varepsilon_{y}(\bar{\nu})}{(\bar{\nu})^{4}} \mathrm{~d} \nu
$$

where $\psi$ represents the orientation dependence of dipole-dipole interaction and in the dynamic averaging limit its value is $(2 / 3)^{\frac{1}{2}}, \phi_{x}$ is the quantum yield of the donor, $n$ is the refractive index of the solvent medium, $N^{\prime}$ the Avogadro number, $f_{x}(\bar{\nu})$ is the spectral distribution of the donor emission measured in quantum and normalized to unity on a wave number $(\bar{\nu})$ scale, $\varepsilon_{y}(\bar{\nu})$ is the spectral distribution of the molar extinction coefficient of the acceptor. Further we have used the emission spectrum of toluene in cyclohexane given by Berlman [15], the absorption spectrum of the solute obtained using UV spectrophotometer (Hitachi), and the values of quantum yield $\phi_{x}$ given by Birks and the value of $R_{0}$ is given in the Table.

In Figure 2 we give the plot of $K_{3}$ versus $D_{x y}$ according to Eq. (9) and deduce the values of $\Lambda$ and $P_{\mathrm{XY}} R_{\text {eff }}$ by least square fit method and these values are given in the Table. From Fig. 2 we notice that the variation of $K_{3}$ with $D_{\mathrm{XY}}$ is linear which shows that $\Lambda$ and $P_{\mathrm{XY}} R_{\mathrm{eff}}$ are independent of temperature as expected according to the compact equation. From the Table we observe that the value of $R_{\mathrm{eff}}=13.6$ $\AA$ deduced by assuming $P_{\mathrm{XY}}$ to be unity is very much larger than $R_{\mathrm{XY}}=7.20 \AA$. But on the other hand the value of $R_{0} / 2=10.6 \AA$ is close to $R_{\text {eff. }}$. This shows that the energy transfer distance between the excited solvent and the solute molecules is much larger than the sum of their molecular radii. It is interesting to note that for neat toluene system [16] $P_{\mathrm{XY}} R_{\mathrm{eff}}$ is $7.74 \AA$ which is very close to $R_{\mathrm{XY}}$ taking $P_{\mathrm{XY}}$ to be unity. This shows that in the pure toluene system the energy transfer is due to close collisions only. Whereas when the diluent such as cyclohexane is added, the energy transfer distance becomes much larger than $R_{\mathrm{xy}}$ indicating that the energy transfer takes place even when the distance between the excited solvent molecule and the solute molecule is very large compared to their molecular radii. This may be understood in the following way.

When an excited solvent molecule is surrounded by a large number of neutral diluent molecules the probability of solvent-solvent energy transfer decreases. Since the viscosity of the medium does not change much by adding the neutral diluent, the diffusion process will not be affected much. Hence solvent-solute energy transfer can compete with migration process even when the solvent-solute distance is large. Therefore, in dilute systems along with the diffusion and migra-

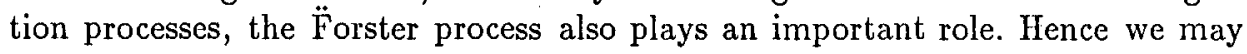
conclude that Förster formulation as adopted by Voltz et al. seems to account for 
the solvent-solute energy transfer in the final step and hence the energy transfer is non-collisional and involves long-range interaction.

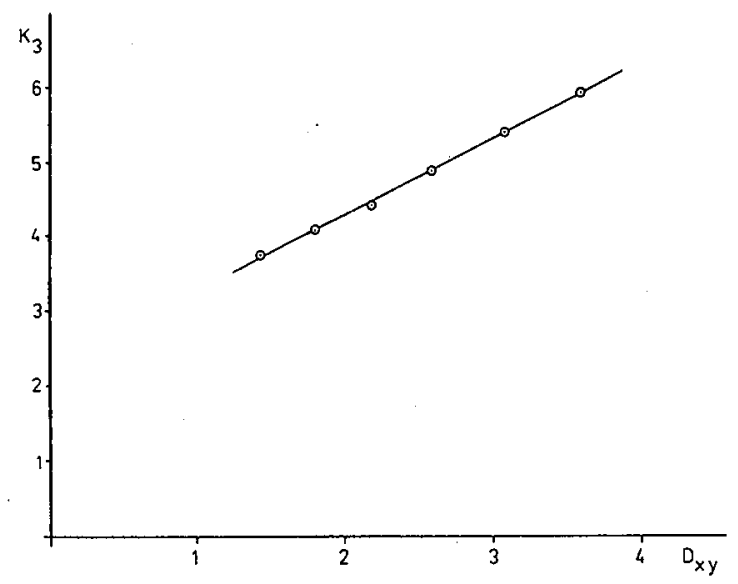

Fig. 2. Variation of $K_{3}$ (in $10^{10}$ mole $^{-1} \mathrm{~s}^{-1}$ ) with $D_{\mathrm{XY}}$ the sum of the self-diffusion coefficients of the solvent and the solute (in $10^{-5} \mathrm{~cm}^{2} \mathrm{~s}^{-1}$ ).

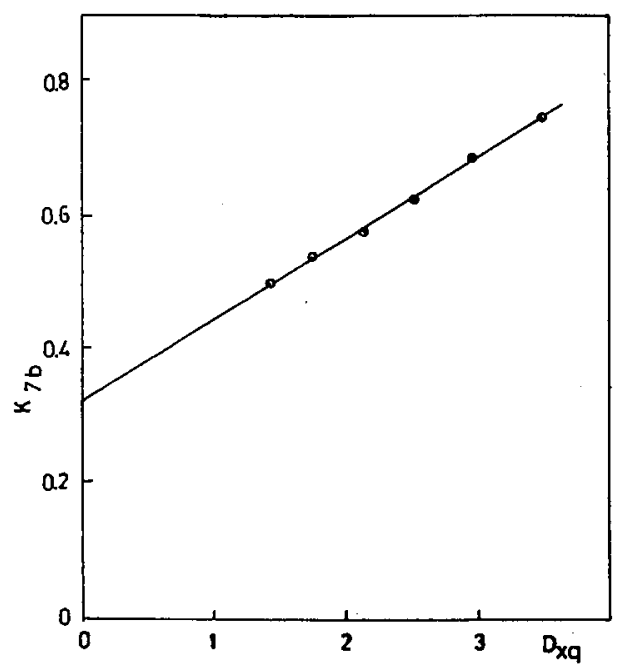

Fig. 3. Variation of $K_{7 \mathrm{~b}}$ (in $10^{10} \mathrm{~mole}^{-1} \mathrm{~s}^{-1}$ ) with $D_{\mathrm{XQ}}$ the sum of the self-diffusion coefficients of the solvent and the quencher (in $10^{-5} \mathrm{~cm}^{2} \mathrm{~s}^{-1}$ ).

Further in Fig. 3 we give the plot of $K_{7 \mathrm{~b}}$ versus $D_{\mathrm{XQ}}$ according to Eq. (9) and deduce the value of $\Lambda$ and $P_{\mathrm{XQ}} R_{\mathrm{eff}}$ by the least square fit method and the values are given in the Table. From Fig. 3 we notice that the variation of $K_{7 b}$ with 
$D_{\mathrm{XQ}}$ is linear which shows that $\Lambda$ and $P_{\mathrm{XQ}} R_{\mathrm{eff}}$ are independent of temperature as in the case of $K_{3}$.

We may now consider the experimental values of $P_{\mathrm{XQ}}$ and $R_{\mathrm{eff}}$. We see from the Table that the value of $P_{\mathrm{XQ}} R_{\text {eff }}$ deduced by using Eq. (9) is equal to $1.59 \AA$ and is smaller than the value of $R_{\mathrm{XQ}}=5.82 \AA$. If we choose $P_{\mathrm{XQ}}$ to be about 0.27 (since $P_{\mathrm{XQ}}$ the probability of quenching per encounter is always less than unity according to [2]) the value of $R_{\text {eff }}$ agrees with that of $R_{\mathrm{XQ}}$. Such a small value of $P_{X Q}$ is also observed by others $[2,19]$. These results evidently show that the quenching process is due to diffusion and migration processes only, with the probability of quenching per encounter $P_{\mathrm{XQ}}$ being less than unity.

\section{Conclusion}

From our experimental results we may conclude that in dilute systems long-range interaction due to Förster process also begins to play a part in the overall solvent-solute energy transfer process whereas quenching process is due to diffusion and migration processes only.

\section{References}

[1] R. Voltz, G. Lausteriat, A. Coche, J. Chem. Phys. 63, 1253 (1966).

[2] J.B. Birks, J.C. Conte, Proc. R. Soc. A 303, 85 (1968).

[3] J.B. Birks, H.Y. Najjar, M.D. Lumb, J. Phys. B 4, 1516 (1971).

[4] A. Chazel, J. Chem. Phys. 67, 4735 (1977).

[5] B. Sipp, R. Voltz, J. Chem. Phys. 79, 434 (1983).

[6] B. Sipp, R. Voltz, J. Chem. Phys. 83, 157 (1985).

[7] B. Sipp, R. Voltz, J. Lumin. 31, 609 (1984).

[8] D.M. Umarjee, G.C. Chikkur, Indian J. Pure Appl. Phys. 22, 351 (1984).

[9] A. Weinreb, in Luminescence of Organic and Inorganic Materials, Eds. H.P. Kallmann, G.M. Spruch, Wiley, New York 1962, p. 44.

[10] D.M. Umarjee, G.C. Chikkur, Phys. Lett. A 112, 57 (1985).

[11] A. Einstein, Investigations on the Theory of Brownian Movement, Dover, New York 1956.

[12] R.D. Mathad, N. Umakantha, Phys. Lett. A 108, 167 (1985).

[13] J.T. Edward, Chem. Ind. (N.Y.) 30, 774 (1956).

[14] T.H. Förster, Ann. Phys. 2, 55 (1948); Z. Nat.forsch. 40, 321 (1949).

[15] I.B. Berlman, Handbook of Fluorescence Spectra of Aromatic Molecules, Academic, New York 1965, p. 47.

[16] S.M. Hanagodimath, G.S. Gadaginamath, G.C. Chikkur, J. Phys. D, Appl. Phys. (communicated).

[17] C. Tanielian, Proc. Int. Conf. Lumin., Hungarian Academy of Sciences, Budapest 1966, p. 468. 